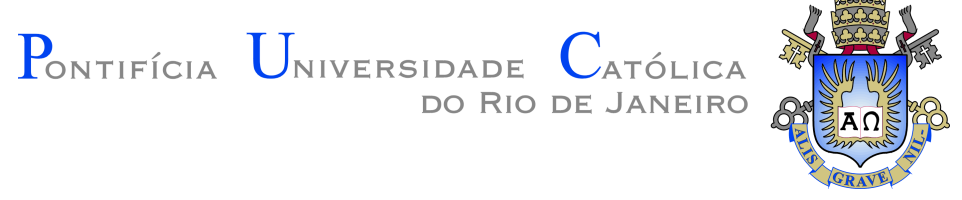

Felipe Jordão Pinheiro de Andrade

LaWie - Deconvolução em picos esparsos usando o LASSO e filtro de Wiener

Dissertação de Mestrado

Dissertação apresentada como requisito parcial para obtenção do grau de Mestre pelo Programa de Pós-graduação em Informática, do Departamento de Informática da PUC-Rio.

Orientador: Prof. Marcelo Gattass

Rio de Janeiro

Setembro de 2020 


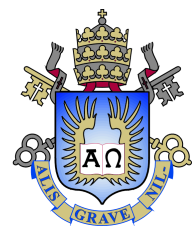

Felipe Jordão Pinheiro de Andrade

\title{
LaWie - Deconvolução em picos esparsos usando o LASSO e filtro de Wiener
}

\begin{abstract}
Dissertação apresentada como requisito parcial para obtenção do grau de Mestre pelo Programa de Pós-graduação em Informática, do Departamento de Informática da PUC-Rio. Aprovada pela Comissão Examinadora abaixo.
\end{abstract}

\author{
Prof. Marcelo Gattass \\ Orientador \\ Departamento de Informática - PUC-Rio
}

Prof. Hélio Côrtes Vieira Lopes

Departamento de Informática - PUC-Rio

Dr. Rodrigo Costa Fernandes

Tecnologia Geofísica - Petrobras

Rio de Janeiro, 25 de Setembro de 2020 
Todos os direitos reservados. É proibida a reprodução total ou parcial do trabalho sem autorização da universidade, do autor e do orientador.

Felipe Jordão Pinheiro de Andrade

Graduou-se em Ciência da Computação pela Universidade Federal do Maranhão. Atualmente faz mestrado no Departamento de Informática da PUC-Rio com enfoque em aprendizado de máquina e renderização. Atualmente trabalha no Instituto Tecgraf em projetos na área de renderização e aprendizado de máquina.

Ficha Catalográfica

Andrade, Felipe Jordão Pinheiro de

LaWie - Deconvolução em picos esparsos usando o LASSO e filtro de Wiener / Felipe Jordão Pinheiro de Andrade; orientador: Marcelo Gattass. - Rio de janeiro: PUC-Rio , Departamento de Informática , 2020.

v., $40 \mathrm{f}$ : il. color. ; $30 \mathrm{~cm}$

Dissertação (mestrado) - Pontifícia Universidade Católica do Rio de Janeiro, Departamento de Informática .

Inclui bibliografia

1. Informática - Teses. 2. Modelagem esparsa;. 3. Deconvolução;. 4. Inversão;. 5. LASSO;. 6. Wiener;. I. Gattass, Marcelo. II. Pontifícia Universidade Católica do Rio de Janeiro. Departamento de Informática . III. Título. 


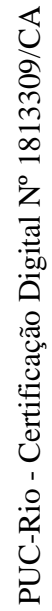

Aos meus pais. 


\section{Agradecimentos}

Agradeço aos meus pais, Tatiana e Eguinaldo, que sempre lutaram para que eu e minha irmã tivessemos a melhor educação possível, ao mesmo tempo que nos deram liberdade para escolher nossos próprios caminhos. Agradeço aos meus avós, Idemar e Walter, e minha irmã, Amanda, que sempre me apoiaram nas minhas decisões que me trouxeram ao mestrado na PUC-Rio. Agradeço minha namorada, Catarina, pelo apoio incondicional e compreenção nessa jornada da pós graduação.

Também agradeço meus colegas do mestrado e do Tecgraf que me apoiaram dando feedbacks para esse trabalho e pelo companherismo nesses ultimos 2 anos.

Agradeço também ao meu professor orientador Marcelo por toda a ajuda e conselhos durante o desenvolvimento deste trabalho.

Por fim, agradeço ao Instituto Tecgraf pelo apoio técnico para o desenvolvimento deste trabalho.

O presente trabalho foi realizado com apoio da Coordenação de Aperfeiçoamento de Pessoal de Nível Superior - Brasil (CAPES) - Código de Financiamento 001. 


\section{Resumo}

Andrade, Felipe Jordão Pinheiro de; Gattass, Marcelo. LaWie Deconvolução em picos esparsos usando o LASSO e filtro de Wiener. Rio de Janeiro, 2020. 40p. Dissertação de Mestrado Departamento de Informática , Pontifícia Universidade Católica do Rio de Janeiro.

Este trabalho propõe um algoritmo para o problema da deconvolução sísmica em picos esparsos. Intitulado LaWie, este algoritmo é baseado na combinação do Least Absolute Shrinkage and Selection Operator (LASSO) e a modelagem de blocos usada no filtro de Wiener. A deconvolução é feita traço a traço para estimar o perfil de refletividade e a wavelet original que deu origem as amplitudes sísmicas. Este trabalho apresenta o resultado do método no dataset sintético do Marmousi2, onde existe um ground truth para comparações objetivas. Além disso, também apresenta os resultados no dataset real Netherlands Offshore F3 Block e mostra a aplicabilidade do algoritmo proposto para não apenas delinear o perfil de refletividades como também para ressaltar características como fraturas neste dado.

\section{Palavras-chave}

Modelagem esparsa; Deconvolução; Inversão; LASSO; Wiener; 


\section{Abstract}

Andrade, Felipe Jordão Pinheiro de; Gattass, Marcelo (Advisor). LaWie - Sparse-Spike Deconvolution with LASSO and Wiener Filter. Rio de Janeiro, 2020. 40p. Dissertação de Mestrado - Departamento de Informática, Pontifícia Universidade Católica do Rio de Janeiro.

This work proposes an algorithm for solving the seismic sparse-spike deconvolution problem. Entitled LaWie, this algorithm is based on the combination of Least Absolute Shrinkage and Selection Operator (LASSO) and the block modeling used in the Wiener filter. Deconvolution is done trace by trace to estimate the reflectivity profile and the convolution wavelet that originated the seismic amplitudes. This work presents the results in the synthetic dataset of Marmousi2, where there is a ground truth for objective comparisons. Also, this work presents the results in a real dataset, Netherlands Offshore F3 Block, and shows the applicability of the proposed algorithm to outline the reflectivity profile and highlight characteristics such as fractures in this data.

\section{Keywords}

Sparse modeling; Deconvolution; Inversion; LASSO; Wiener; 


\section{Sumário}

1 Introdução $\quad 11$

1.1 Objetivo Geral 11

1.2 Objetivos Específicos 11

$\begin{array}{lll}1.3 & \text { Organização da Dissertação } & 12\end{array}$

2 Revisão Bibliográfica $\quad 13$

2.1 Deconvolução Sísmica 13

3 Método Proposto $\quad 16$

3.1 Deconvolução Sísmica 16

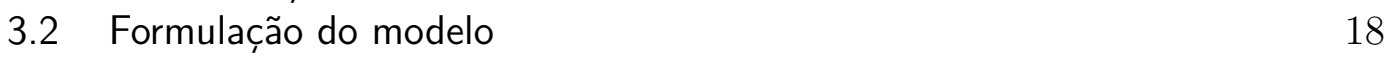

3.2.1 Estimação do perfil de Refletividades 19

3.2.2 Estimação da Wavelet 20

$\begin{array}{lll}3.3 \text { Algoritmo } & 21\end{array}$

$4 \quad$ Medidas de Similaridade e de Erro $\quad 23$

4.1 Cálculo da Similaridade entre Wavelets 23

4.2 Cálculo da Similaridade Entre os Traços de Refletividade 24

4.3 Medida de Erro Quadrática 24

5 Resultados $\quad 26$

$\begin{array}{lll}5.1 & \text { Testes } & 26\end{array}$

5.1.1 Dado Sintético - Marmousi2 27

5.2 Dado Real - Netherlands Offshore F3 Block 30

$\begin{array}{ll}5.3 \text { Discussão } & 31\end{array}$

6 Conclusões e Trabalhos Futuros $\quad 35$

6.1 Trabalhos Futuros 36

$\begin{array}{ll}\text { Referências bibliográficas } & 38\end{array}$ 


\section{Lista de figuras}

$\begin{array}{lll}\text { Figura 3.1 Exemplo do processo de convolução. } & 17\end{array}$

Figura 4.1 Problemas encontrados ao medir a similaridade do cosseno entre as wavelets.

Figura 4.2 Visualização do problema ao calcular a similaridade do cosseno entre duas curvas que estão deslocadas.

Figura 5.1 Perfil de refletividades calculado.

Figura 5.2 Amplitudes sísmicas obtidas pela convolução do perfil de refletividades com uma wavelet Ricker de frequência igual a $30 \mathrm{~Hz}$.

Figura 5.3 Resultados apresentados do teste $1 . \quad 30$

Figura 5.4 Resultados apresentados do teste 2. 30

Figura 5.5 Resultados apresentados do teste 3. 31

Figura 5.6 Resultados da deconvolução da inline 260 do F3. 32

Figura 5.7 Figura ressaltando as descontinuidades criadas por falhas sísmicas que podem ser observadas mais claramente no perfil de refletividades estimado.

Figura 5.8 Gráfico da medida de erro por iteração. 34 


\section{Lista de tabelas}

$\begin{array}{lll}\text { Tabela 5.1 } & \text { Parâmetros teste } 1 . & 29\end{array}$

Tabela 5.2 Parâmetros teste $2 . \quad 29$

Tabela 5.3 Parâmetros teste $3 . \quad 29$

$\begin{array}{lll}\text { Tabela 5.4 Resultados de similaridade calculados. } & 29\end{array}$

Tabela 5.5 Comparação do tempo de execução da deconvolução em sequencial e paralelo 34 


\section{Introdução}

A Geofísica Computacional busca desenvolver algoritmos e procedimentos que facilitem o trabalho dos geólogos e geofísicos na interpretação do modelo geológico da subsuperfície da terra. Esses modelos são fundamentais na exploração e produção eficiente e segura de hidrocarbonetos.

Dentre os algoritmos da Geofísica Computacional, cabe destacar os algoritmos de deconvolução e de inversão sísmica. Estes algoritmos buscam a partir do perfil de amplitude sísmica, separar a onda impulsiva da fonte das refletividades da subsuperfície. Essas refletividades são importantes para compreensão das camadas litológicas presentes na subsuperfície da Terra.

No programa de pós-graduação do Departamento de Informática da PUC-Rio, o problema da Deconvolução Sísmica em Picos Esparsos de Refletividade é uma linha de pesquisa que já produziu uma tese de Doutorado e alguns trabalhos [Fernandes, 2019] [Fernandes, Lopes e Gattass, 2017] [Andrade, Gattass e Fernandes, 2020]. Este trabalho se insere dentro desta linha de pesquisa e avança o trabalho de Fernandes [Fernandes, 2019] em dois pontos importantes. O primeiro é na modificação do algoritmo proposto por Fernandes para incluir a modelagem da onda impulsiva da fonte com base no filtro em bloco de Wiener. O segundo é uma nova métrica para medir distância entre vetores esparsos mais apropriada para o problema em questão. Estes dois avanços são as principais contribuições desta dissertação.

\section{1}

\section{Objetivo Geral}

O objetivo central desta dissertação é apresentar uma metodologia que, dada uma matriz de amplitudes resultante de um levantamento sísmico, consiga com base no modelo convolucional, separar a componente das refletividades da onda geradora.

\section{2}

\section{Objetivos Específicos}

Essa dissertação tem por objetivos testar as seguintes hipóteses: 
- Estimar simultaneamente as refletividades e a onda geradora de um perfil de amplitudes sísmicas, com base na minimização do erro de reconstrução e na maximização da esparsidade do vetor de refletividades.

- Fazer a estimação por traço do componente de refletividades levando em consideração a intensidade e a amplitude dos picos.

- Estimar a onda geradora relacionada ao perfil de amplitudes incluindo ondas com mudança de fase.

- Propor uma forma mais eficaz de avaliação da qualidade da deconvolução que se baseie na similaridade do cosseno e seja robusta face a pequenos deslocamentos de fase.

- Avaliar uma forma simples de paralelismo no algoritmo de deconvolução.

\section{3}

\section{Organização da Dissertação}

Esta dissertação está organizada em cinco capítulos, sendo o primeiro esta introdução. O Capítulo 2 apresenta a revisão bibliográfica das áreas de deconvolução e modelagem esparsa usadas como base para o desenvolvimento deste trabalho. O capítulo 3 apresenta a modelagem algébrica do problema detalhando as etapas de estimação de cada um dos dois componentes. Ele também apresenta o método proposto com um pseudo código. O capítulo 4 detalha uma nova proposta de medida de erro para medir a qualidade do processo de deconvolução. O capítulo 5 mostra os testes propostos, usando dados sintéticos e dados reais. Este capítulo discute também os resultados obtidos e os parâmetros usados. Por fim, o Capítulo 6 faz as considerações finais deste trabalho e apresenta propostas para trabalhos futuros. 


\section{2}

\section{Revisão Bibliográfica}

Neste capítulo serão discutidas as referências usadas como base para o desenvolvimento deste trabalho. Primeiramente, são comentadas as referências significativas ao problema de deconvolução, com o objetivo de entender o contexto que esse trabalho se insere. Em seguida, as referências relacionadas ao método, que usa conceitos de modelagem esparsa e aprendizado por dicionário, são comentadas.

\section{1}

\section{Deconvolução Sísmica}

O problema da deconvolução sísmica é antigo dentro da área da geofísica, com referências datando da década de 70. No trabalho de [Kormylo e Mendel, 1978], uma das referências relacionadas mais antigas, os autores usam uma função probabilística para achar a amplitude dos refletores sísmicos, que apesar de não modelar o problema como uma deconvolução sísmica, também foca no problema de recuperação do perfil de refletividades como este trabalho.

Com o passar do tempo, o problema da deconvolução sísmica tem sido abordado por alguns métodos diferentes dentro da literatura na área da geofísica. Em [Velis, 2008], um método estocástico é usado para achar a amplitude dos refletores sísmicos, ja em [Kormylo e Mendel, 1978] e [Kaaresen e Taxt, 1998] os autores modelam os picos de refletividade como uma função probabilistica. Porém, é notável a maioria dos trabalhos recentes voltados para deconvolução sísmica propõem uma metodologia que busca otimizar em cima de uma norma reforçando a característica esparsa, e desejável, do vetor de refletividades.

O trabalho de [Wang et al., 2016] foi uma das bases mais importantes para o desenvolvimento desta dissertação. Neste trabalho, o autor usa o treinamento de um dicionário para fazer o processo da deconvolução, onde este dicionário é representado por uma matriz de Toeplitz representando a wavelet geradora. O uso da matriz de Toeplitz como um dicionário também foi usado anteriormente por [Kibangou e Favier, 2007]. O autor ressalta que é ideal usar essa representação da wavelet para aproveitar das técnicas de fatoração rápida 
para essa matriz específica.

Wang propõe que os dois componentes, wavelet e perfil de refletividades, sejam invertidos simultaneamente, usando um conjunto de traços por vez. Esse processo se dá pela etapa da estimação da refletividade enquanto a wavelet é fixa e, após a estimação das refletividades deste conjunto de traços, a wavelet é estimada enquanto o componente das refletividades é fixo. Para estimar as refletividades, os autores usaram o algoritmo Fast Iterative Shrinkagethresholding (FISTA)[Beck e Teboulle, 2009], enquanto que para estimar a wavelet do conjunto de traços dado como entrada, é usado o algoritmo do Fused Lasso.

Em [Fernandes, Lopes e Gattass, 2017], os autores propuseram uma abordagem também baseada no trabalho de Wang, porém com uma modificação que o valor do parâmetro relacionado a esparsidade do vetor não precise ser dado como entrada, apênas o número de picos desejados, enquanto o algoritmo usa uma busca binária para encontrar um valor ideal do parâmetro da esparsidade. Além disso, propuseram também uma forma de avaliar a qualidade da deconvolução quando existe um ground truth para uma comparação direta.

Recentemente, alguns autores têm considerando um comportamento não estacionário da wavelet para melhor estimar os refletores recuperados. Em [Fernandes, 2019], os autores aplicaram uma técnica de aumento artificial de dicionário para melhor estimar as variações na wavelet no tempo. Em [Sui e Jianwei, 2019] os autores estenderam o trabalho de Wang levando em consideração que a wavelet não tem um comportamento estacionário. Em outras palavras, tentam modelar os efeitos de atenuação e dispersão na wavelet para melhor estimar o perfil de refletividades. Para isso usam a expressão de uma convolução não estacionária [Margrave e Henley, 2011] que ajusta o valor de um parâmetro Q modificando a característica da matriz de toeplitz que representa a wavelet.

\section{Modelagem Esparsa e Recuperação do Sinal Esparso}

Como mencionado anteriormente, para a tarefa deconvolução, muitos trabalhos otimizam uma certa norma que reforce a característica de esparsidade do vetor. Porém, o motivo de tal interesse está diretamente relacionado às simples formulações matemáticas que permitem uma computação eficiente dos processos deconvolucionais em parceria com o rico campo da modelagem esparsa [Rish e Grabarnik, 2015] [Dumitrescu e Irofti, 2018].

Este trabalho se baseia na teoria de recuperação do sinal esparso que [Rish e Grabarnik, 2015] e [Elad, 2010] descrevem em ambos os livros. Essencialmente, o problema gira em torno de achar a minima cardinalizade que 
restringe algum problema de otimização. Essa cardinalidade é a quantidade de elementos não zero é interpretada como a norma $l_{o}$ do vetor solução. Como o problema de achar a menor cardinalidade do vetor solução é conhecido por ser NP-Difícil, é comum uma aproximação ser feita para garantir eficiência computacional, reescrevendo o problema para uma programação linear otimizando a norma $l_{1}$.

Com a transformação deste problema para resolver a norma $l_{1}$, existem vários algoritmo conhecidos na literatura que são capazes solucionar o problema em um tempo razoável. Dentre eles, é destacado o LASSO [Tibshirani, 1996] usado neste trabalho. Segundo Tibshirani, o Least Absolute Shrinkage and Selection Operator (LASSO) é capaz de minimizar a soma residual dos quadrados, sujeito que a soma do valor absoluto dos coeficientes seja menor que uma constante. 


\section{3}

\section{Método Proposto}

Este capítulo aborda o problema de deconvolução em imagens sísmicas. Primeiramente, é apresentado a modelagem matemática, deste fenômeno físico, tendo em vista as características de uma onda e como ela se relaciona com o substrato que percorre. Também é comentado como o problema da deconvolução sísmica é um problema de inversão aproximado e por isso imprecisões são esperadas.

Por fim, neste capítulo, é descrito, uma proposta de deconvolução em picos esparsos de refletividade. É apresentada a formulação desse problema dentro da teoria de minimização do quadrado do erro, como é estimado o componente da onda e o componente das refletividades individualmente e por fim é apresentado o pseudo-código que representa o processo proposto.

\section{1}

\section{Deconvolução Sísmica}

Segundo [Rosa, 2010], um método de inversão sísmica é a aplicação de processos com a finalidade de estimar as propriedades elásticas que deram origem aos dados registrados. Se for levado em consideração o modelo matemático mais simples que representa o traço sísmico, as propriedades elásticas às quais Rosa se refere são a assinatura da fonte ou pulso sísmico, e uma série de coeficientes de reflexão. Esse modelo matemático é chamado de modelo convolucional do traço sísmico para incidência vertical e é descrito pela seguinte equação:

$$
s(t)=p(t) * r(t)+n(t)
$$

Onde o símbolo " $*$ " representa a operação da convolução, $p(t)$ é o pulso sísmico, $r(t)$ é a série de coeficientes de reflexão e $n(t)$ é um ruído associado ao traço. Todas as variáveis em função do tempo t.

Na Figura 3.1, observa-se um exemplo do resultado de um processo de convolução de uma wavelet estacionária com um perfil de refletividades para gerar a amplitude sísmica de um traço. É separada a contribuição de cada pico de refletividade convolvido com a wavelet para mostrar a contribuição de cada um para a amplitude do traço sísmico final. Dessa forma é possível mostrar como os sinais se misturam e, consequentemente, problema se torna 
mais complexo.
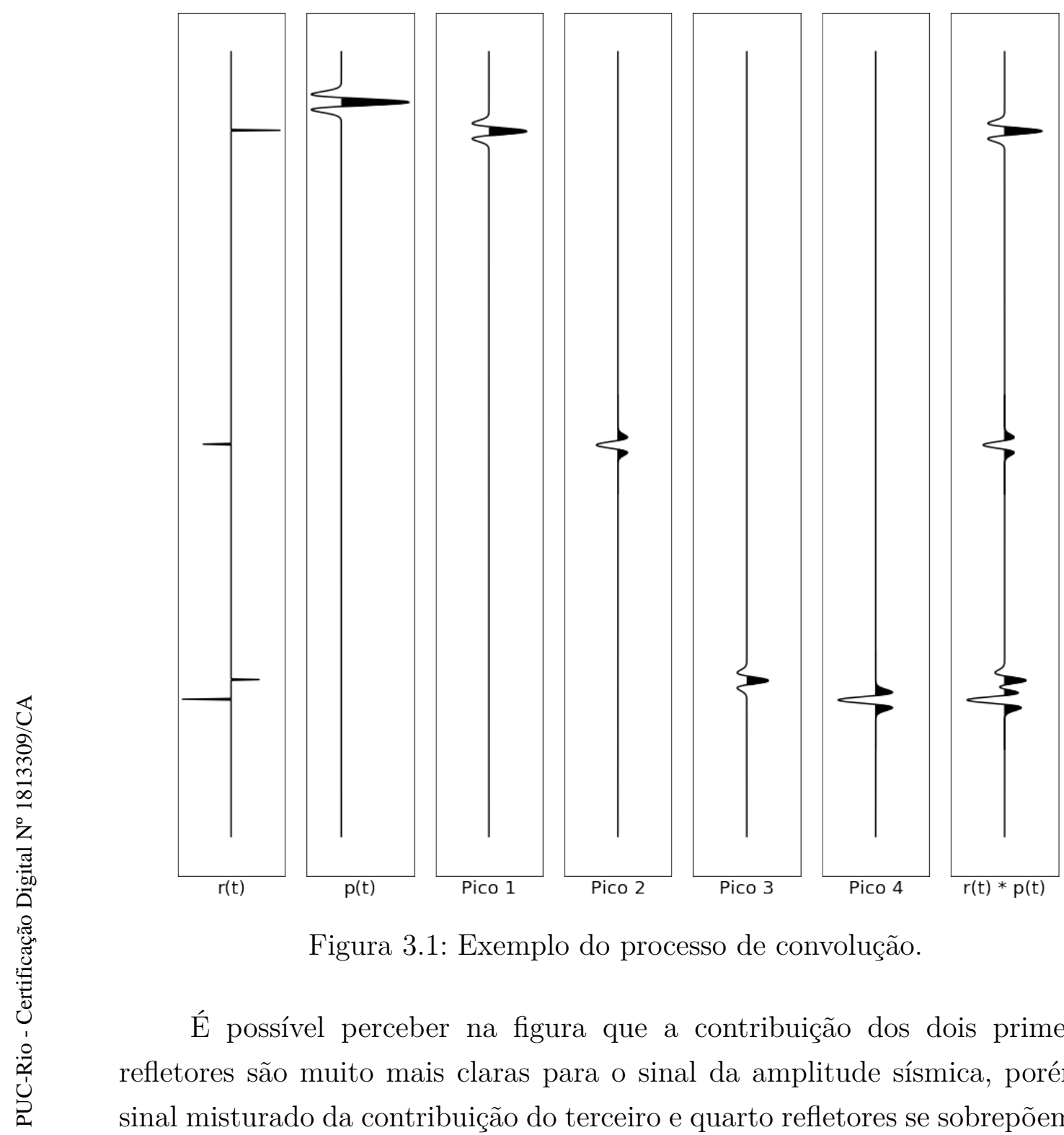

Figura 3.1: Exemplo do processo de convolução.

É possível perceber na figura que a contribuição dos dois primeiros refletores são muito mais claras para o sinal da amplitude sísmica, porém o sinal misturado da contribuição do terceiro e quarto refletores se sobrepõem de forma que não é mais tão fácil interpretar a forma do pulso gerador e os picos de refletividade que criaram aquele sinal. Será discutido adiante que um dos problemas que os métodos de deconvolução sísmica enfrentam é a separação dos dois componentes que formam a amplitude sísmica, quando os refletores são muito próximos.

Dentro dessa definição da deconvolução sísmica de picos esparsos, levamos em consideração a qualidade de quatro características para avaliar o método [Fernandes, Lopes e Gattass, 2017] [Velis, 2008].

- A quantidade dos picos extraídos que mostra a capacidade do método de estimar uma quantidade correta de picos para cada traço.

- A posição desses picos, pois essa característica é traduzida para a continuidade lateral dos refletores, facilitando o trabalho da interpretação. 
- A magnitude dos picos estimados, pois elas representam as características físicas da onda fazendo a transição do meio de propagação.

- A fase do pulso sísmico, que está diretamente correlacionada a variação positiva ou negativa dos coeficientes de refletividade causada pela mudança de densidade do meio de propagação.

\section{2}

\section{Formulação do modelo}

Nesta seção será apresentada como foi formulada a solução proposta para o problema da deconvolução sísmica em picos esparsos de refletividade, onde a principal característica que queremos reforçar é que o vetor de refletividades seja esparso.

Considerando uma matriz $S$ que armazena a amplitude de uma seção sísmica 2D e que $R$ é uma matriz de refletividades que armazena a variação da impedância sísmica pelo tempo de reflexão da onda. Essas duas matrizes têm dimensão de $m \times n$, onde $m$ é o numero de traços e $n$ é o número de amostras de tempo do traço.

Considerando também que $s$ é um vetor de tamanho $n \times 1$ representando a amplitude de um dos traços sísmicos. $W$ é uma matriz de Toeplitz da wavelet geradora e $r$ é um vetor $n \times 1$ esparso representando o perfil de refletividades de um traço na escala do tempo.

O objetivo de deconvolução sísmica em picos esparsos consiste em encontrar um vetor de refletividade esparso, $r$, que minimize:

$$
\alpha=\min _{W, r}\|s-W r\|_{F}^{2}
$$

em [Wang et al., 2016], $W$ é uma matriz Toeplitz $n \times n$ da wavelet de origem, dada por:

$$
\begin{gathered}
W=\left[\begin{array}{cccccccc}
w_{0} & w_{-1} & w_{-2} & \cdots & w_{-b} & 0 & \cdots & 0 \\
w_{1} & w_{0} & w_{-1} & \cdots & w_{-b} & 0 & \cdots & 0 \\
w_{2} & w_{1} & w_{0} & \cdots & w_{-b} & 0 & \cdots & 0 \\
\vdots & \vdots & \vdots & \vdots & \vdots & \vdots & \vdots & \vdots \\
0 & \cdots & w_{b} & w_{b-1} & \cdots & w_{1} & w_{0} & w_{-1} \\
0 & \cdots & 0 & w_{b} & w_{b-1} & \cdots & w_{1} & w_{0}
\end{array}\right] \\
w=\left[\begin{array}{lllllll}
w_{-b} & \cdots & w_{-1} & w_{0} & w_{1} & \cdots & w_{b}
\end{array}\right]
\end{gathered}
$$

Onde $w$ tem um número de elementos igual a $2 b+1$ e é a representação discreta da wavelet geradora da sísmica.

Em álgebra linear, esparsidade está diretamente relacionada a minimização $\ell_{0}$, pois a pseudo norma zero de um vetor pode ser transcrita como a 
quantidade de elementos não nulos. Porém essa classe de problemas é conhecida por ter uma característica combinatorial e consequentemente NP-Difícil. Em outras palavras, não é conhecido um algoritmo que resolva esse problema com uma complexidade polinomial.

Existem soluções de busca em subespaços que resolvem aproximadamente problemas NP-Difíceis. O trabalho de [Velis, 2008] é um exemplo desta abordagem, porém dado o esforço computacional e a dificuldade de prova de convergência, a maioria dos autores escolhe um caminho distinto pra elaborar uma solução para o problema da minimização de $\ell_{0}$.

Em vez de propor uma solução heurística para um problema combinatorial, o caminho tomado foi relaxar o problema da regularização $\ell_{0}$ e resolver um problema de regularização $\ell_{1}$. A grande vantagem dessa mudança na definição do problema é que a regularização $\ell_{1}$ torna o problema convexo, quadrático e com diversos algoritmos de otimização conhecido que conseguem resolve-lo eficientemente, além de ainda reforçar o característica de esparsidade desejada.

Após relaxar o problema, chegamos na equação 3-3:

$$
\alpha=\min _{W, r} \frac{1}{2}\|s-W r\|_{F}^{2}+\lambda \sum_{i=1}^{n}\|r\|_{1}
$$

Essa equação deixa claro a função objetivo e mostra que deve-se estimar a matriz $W$ e o vetor $r$ para calcular o erro sobre o vetor $s$. Também pode-se notar que o problema na equação 3-3 não é convexo para todas as variáveis do problema.

É comum que métodos que resolvem o problema de Deconvolulção em Picos Esparsos, a estimação de uma variável por vez. Isso é feito para simplificar o problema principal em subproblemas menores e com a característica convexa. Nesta dissertação, similarmente, é feita a estimativa de cada variável separadamente. Na subseção 3.2.1 apresenta-se a forma de se estimar a variável $r$ das refletividades usando a otimização do Lasso, enquanto na 3.2.2 apresenta-se a formulação para o processo de estimação da variável $W$ usando os princípios da modelagem por blocos de Wiener e a resolução do sistema linear usando mínimos quadrados.

\subsection{1}

\section{Estimação do perfil de Refletividades}

Para a etapa de estimação das refletividades, a variável $W$ é fixa e o subproblema a ser resolvido, é o de se estimar a variável $r$. Para alcançar esse objetivo, impõe-se a regularização $\ell_{1}$ sobre a variável $r$ para obter uma solução esparsa, ao mesmo tempo que é usada a matriz de Toeplitz $W$. O problema descrito na equação 3-4 é resolvido para cada traço que compõe $S$. 


$$
\alpha=\min _{r_{i}} \frac{1}{2}\left\|s_{i}-W r_{i}\right\|_{2}^{2}+\lambda\left\|r_{i}\right\|_{1}
$$

Onde, $\mathrm{s}_{i}$ é a coluna i de $S$ e $r_{i}$ é o traço i do perfil de refletividades $R$. Neste trabalho, para resolver esse subproblema foi escolhido o algoritmo do LASSO.

\subsection{2}

\section{Estimação da Wavelet}

Na segunda etapa, para cada traço $i$, dado a amplitude $s_{i}$ e a refletividade $r_{i}$, estimada no passo anterior, é feita a estimação de $w_{i}$ usando a modelagem por blocos de Wiener. A wavelet final é a média aritimética simples de todas as wavelets estimadas por traço.

Em 1949, Norbert Wiener formulou uma teoria que constitui a base de filtros de mínimos quadrados lineares dependentes de dados em muitas áreas. Os coeficientes de um filtro de Wiener buscam minimizar a distância quadrática média entre a saída do filtro e o sinal desejado, assim como o problema inverso descrito acima [Wiener, 1964]. A teoria de Wiener original pressupõe que os sinais são processos estacionários, no entanto, se periodicamente recalcularmos os coeficientes do filtro para cada bloco de $\mathrm{N}$ amostras de sinal, o filtro se adapta às características médias dos sinais dentro dos blocos e torna-se adaptativo de bloco. Este método usa esta versão em bloco.

A abordagem de blocos de Wiener no contexto do nosso problema começa com uma estimação do erro dada por:

$$
e_{i}=s_{i}-\tilde{\mathbf{R}} w_{i}
$$

Onde,

é uma matriz $n \times l$.

$$
\tilde{R}=\left[\begin{array}{ccccc}
r_{1} & 0 & \cdots & 0 & 0 \\
r_{2} & r_{1} & & \vdots & \vdots \\
r_{3} & r_{2} & \cdots & 0 & 0 \\
\vdots & r_{3} & \cdots & r_{1} & 0 \\
r_{m-1} & \vdots & \ddots & r_{2} & r_{1} \\
r_{m} & r_{m-1} & \ddots & \vdots & r_{2} \\
0 & r_{m} & \cdots & r_{m-2} & \vdots \\
0 & 0 & \cdots & r_{m-1} & r_{m-2} \\
0 & 0 & & r_{m} & r_{m-1} \\
0 & 0 & 0 & \cdots & r_{m}
\end{array}\right]
$$

Para minimizar esse erro usando a teoria dos minimos quadrados, computamos a forma quadrática de: 


$$
\min \left(e^{T} e\right)
$$

Onde:

$$
e^{T} e=\left(s_{i}-\tilde{\mathbf{R}} w_{i}\right)^{T}\left(s_{i}-\tilde{\mathbf{R}} w_{i}\right)
$$

Expandindo esse produto:

$$
e^{T} e=s_{i}^{T} s_{i}-s_{i}^{T} \tilde{\mathbf{R}} w_{i}-\left(\tilde{\mathbf{R}} w_{i}\right)^{T} s_{i}+\left(\tilde{\mathbf{R}} w_{i}\right)^{T} \tilde{\mathbf{R}} w_{i}
$$

Derivando com relação a $w_{i}$, e igualando a zero:

$$
\frac{\partial e^{T} e}{\partial w_{i}}=-2 \tilde{\mathbf{R}}^{T} s_{i}+2 \tilde{\mathbf{R}}^{T} \tilde{\mathbf{R}} w_{i}=0
$$

Chegamos ao sistema de equações $l \times l$ a seguir:

$$
\tilde{\mathbf{R}}^{T} \tilde{\mathbf{R}} w_{i}=\tilde{\mathbf{R}}^{T} s_{i}
$$

Com o sistema de equações acima é possível fazer a estimação da variável $w_{i}$ usando um método de resolução de sistemas lineares. Foi escolhido o método dos mínimos quadrados, pela simplicidade e nenhuma parametrização necessária.

\section{3}

\section{Algoritmo}

O algoritmo do processo de deconvolução proposto, como dito anteriormente, tem como entrada uma imagem sísmica gerada por um processo convolucional e uma wavelet inicial que serve como ponto de partida para a estimação da wavelet associada a sísmica. O algoritmo executa usando as informações de cada traço por vez, executando os dois processos mencionados em 3.2.1 e 3.2.2. Em outras palavras, o algoritmo faz um loop por todos os traços estimando o perfil de refletividade e a wavelet associada a esse traço. A implementação do LASSO usada está disponível na biblioteca python Scikit-learn [Pedregosa et al., 2009] enquanto a implementação usada para a resolução do sistema linear usando mínimos quadrados esta disponível na biblioteca python Numpy [Walt e Gael, 2011].

Após esse loop, faz a atualização da wavelet para a próxima iteração do algoritmo. A atualização corresponde a fazer a média entre as wavelets estimadas para cada traço como na linha 7 e atualizar a matriz de Toeplitz para a próxima iteração do algoritmo. O algoritmo executa esse processo 
iterativamente até o critério de parada ser alcançado.

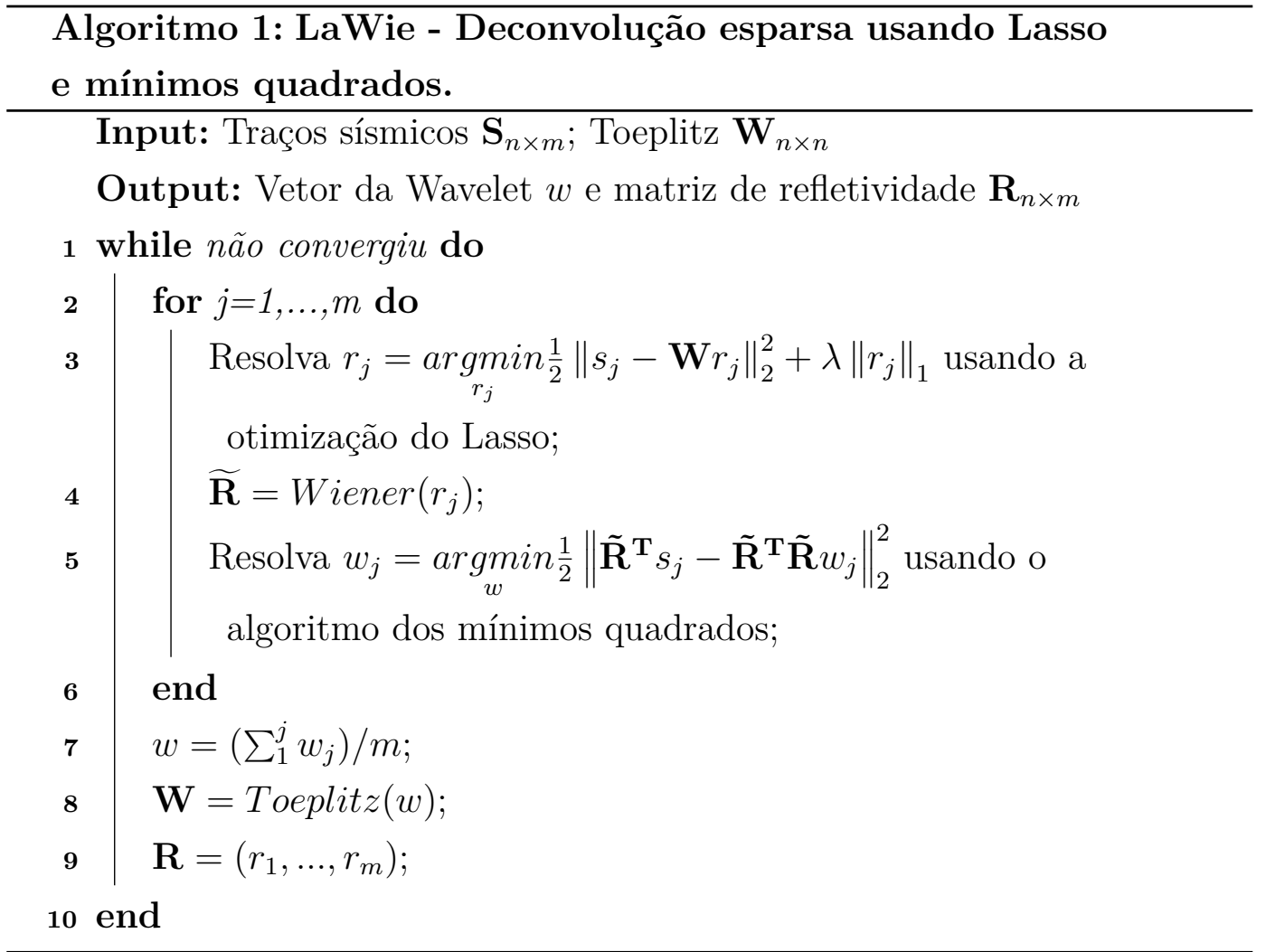

Por fim, tendo em vista a característica do método de fazer a deconvolução por traço de forma independente, o algoritmo foi adaptado para executar em paralelo em CPU. Por causa desta característica, é possivel fazer a instanciação de várias threads de processamento que são distribuidas para os núcleos da CPU e podem ser executadas concorrentemente. Em vez de executar sequencialmente o loop da linha 2 do Algoritmo 1, esse loop foi paralelizado. Para auxiliar esse processo, a biblioteca python Joblib [Varoquaux e Grisel 2009] foi usada. Esta mudança implica em uma diminuição significativa do tempo de processamento que é analisada em mais detalhes na seção de discussão no Capítulo 5. 


\section{4}

\section{Medidas de Similaridade e de Erro}

Para avaliar a qualidade do método proposto no capítulo anterior, é importante conseguirmos medir o quão aproximada a resposta obtida está da resposta real. Por essa razão, esse capítulo tem o objetivo de apresentar as medidas de similaridade usadas para comparar a wavelet estimada com a original, assim como a similaridade calculada para o perfil de refletividades estimado e o original.

Além das medidas de similaridade propostas, é usada uma medida de cálculo do erro quando não temos a resposta dos componentes de refletividades e wavelet originais para fazer uma comparação. Desta forma, a medida de erro reflete se a solução estimada se aproxima, ou se distancia da amplitude dada como entrada.

\section{1}

\section{Cálculo da Similaridade entre Wavelets}

O cálculo da similaridade, entre a wavelet estimada pelo método de deconvolução proposto e a wavelet original responsável pela criação do perfil de amplitude, é necessária como um método quantitativo de avaliação.

Para avaliar a proximidade entre essas duas curvas, este trabalho usa a similaridade do cosseno dado pela Equação 4-1. A medida é representada dentro do intervalo $[-1,+1]$, sendo +1 valores iguais, -1 valores opostos e 0 vetores ortogonais.

$$
C S=\frac{\langle u, v\rangle}{\|u\|_{2}\|v\|_{2}}
$$

Porém, é comum que a wavelet estimada tenha a mesma forma que a wavelet original, mas não estejam precisamente sobrepostas. Isso pode acontecer por uma limitação do método de deconvolução ou até porque o comprimento da wavelet original e da inicial são distintos, como na Figura 4.1. Por esse motivo o cálculo da similaridade entre duas wavelets foi feito deslocando uma delas e calculando a similaridade do cosseno. Dessa forma a métrica reportada é igual à maior similaridade encontrada acompanhada do deslocamento necessário para obtenção deste valor. 


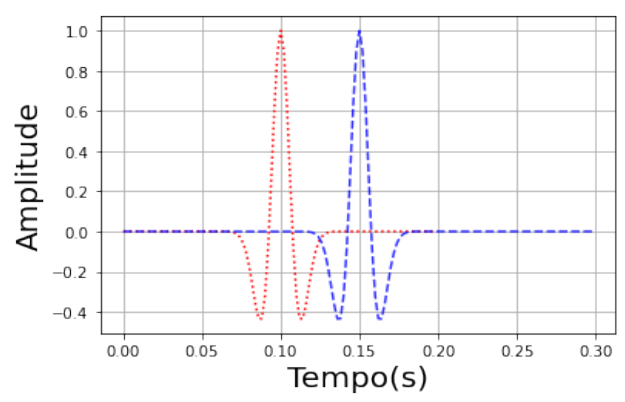

(a) Comparação entre duas curvas de comprimento distinto.

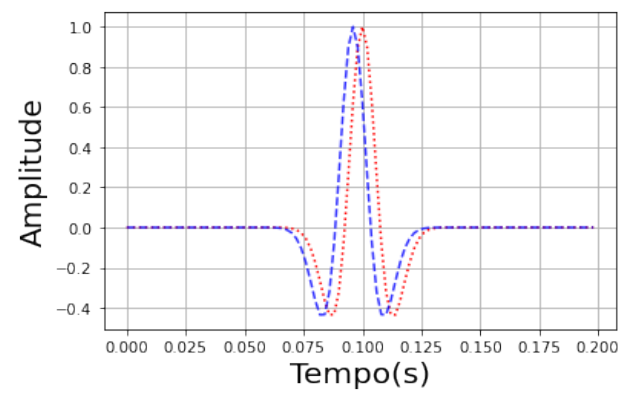

(b) Comparação entre as curvas deslocadas horizontalmente.

Figura 4.1: Problemas encontrados ao medir a similaridade do cosseno entre as wavelets.

\section{2}

\section{Cálculo da Similaridade Entre os Traços de Refletividade}

Para o cálculo da similaridade entre o perfil de refletividades estimado e o original, este trabalho usa a medida da similaridade do cosseno, que também é usado em outros trabalhos para este mesmo fim [Fernandes, Lopes e Gattass, 2017], porém com uma variação para melhor tratar a comparação entre vetores esparsos.

Como no cálculo da similaridade entre duas wavelets na seção anterior, o perfil de refletividades de um traço pode não estar perfeitamente alinhado, fazendo com que a medição usando a Equação 4-1 não represente de forma acurada a similaridade entre as refletividades do traço. Essa discrepância se torna mais evidente em um vetor esparso. Na Figura 4.2 podemos observar o problema e o impacto na medida da similaridade do cosseno.

Para resolver esse problema, é feito a transformação do vetor de refletividades esparso para um vetor denso, fazendo a convolucão com uma curva gaussiana. O resultado desse processo e a medida de similaridade calculada pode ser observada na Figura 4.2.

\section{3}

\section{Medida de Erro Quadrática}

Este trabalho também usa uma medida de erro quadrática para estimar o quão distante a solução encontrada está das amplitudes de entrada, quando não existe a informação dos componentes reais para fazer uma análise mais precisa usando as medidas apresentadas anteriormente. A medida de erro é dada pela Equação 4-2.

$$
E=\|S-W \cdot R\|^{2}
$$




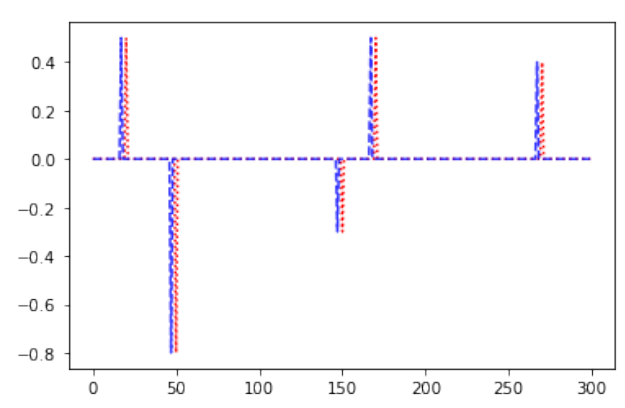

(a) Vetor esparso representando a resposta da refletividade de um traço. A similaridade do cosseno para esses dois vetores esparsos é igual a zero porquê não estão exatamente sobrepostos.

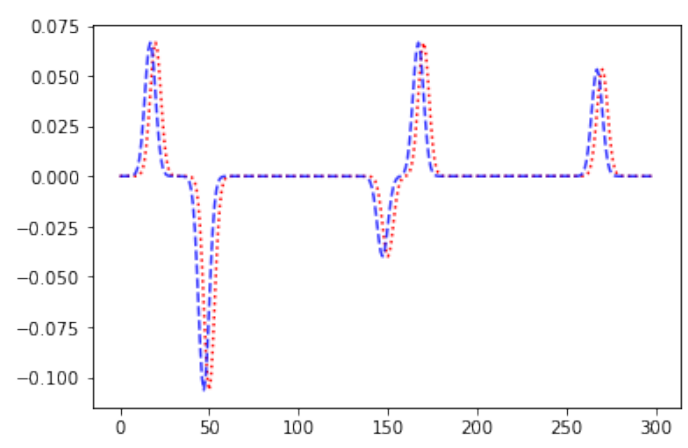

(b) Traço convolvido com gaussiana ( $\sigma=$ 3). A similaridade do cosseno para esses dois vetores densos é de 0.7787 .

Figura 4.2: Visualização do problema ao calcular a similaridade do cosseno entre duas curvas que estão deslocadas.

Onde, $S_{n \times m}$ é a matriz de amplitudes sísmicas, $W_{n \times n}$ é a matriz Toeplitz representando a Wavelet, e $R_{n \times m}$ são as refletividades estimadas. 


\section{Resultados}

Este capítulo expõe os resultados da aplicação do método de deconvolução em picos esparsos descrito no capítulo anterior. Primeiramente, é feita a descrição das condições e parâmetros de cada teste, em seguida é feita a comparação e discussão dos resultados apresentados.

\section{1}

\section{Testes}

Os testes foram propostos com objetivo observar a capacidade do método de realizar a deconvolução sísmica. Dois cenários de teste foram propostos:

1. Cenário controlado: Usa um dado sintético, onde se conhecem as todas as variáveis do problema e é possível fazer uma comparação dos componentes estimados e os componentes reais. Esse teste é importante para reconhecer a capacidade do método em um cenário próximo do real onde podemos fazer o cálculos de similaridade entre os componentes estimados e os reais.

2. Cenário real: Usa um dado real de amplitudes sísmicas. Representa o tipo de informação que os geólogos e geofísicos tem disponíveis para analisar um campo. Este cenário tem por objetivo avaliar o método proposto em um cenário cego, onde não se conhece as variáveis.

Para os testes no dado sintético, as medidas de similaridade do perfil de refletividades e da wavelet estimada serão reportados como descrito no Capítulo 4. Além disso é reportado o tempo de execução para o teste sequencial e em paralelo. Para o cenário real a análise tem um caráter mais subjetivo, tendo em vista que os componentes de refletividades e wavelet geradora não estão disponíveis para uma comparação, dependendo de uma análise feita por um especialista. Para esse teste também é reportado tempo de execução sequencial e paralelo. 


\subsection{1}

\section{Dado Sintético - Marmousi2}

Para a primeira bateria de testes, o dado sintético do Marmousi2 [Martin, Wiley e Marfurt, 2006] foi usado. A escolha desse dado foi por causa do uso do mesmo para diversos outros trabalhos dentro da literatura de deconvolução em picos esparsos de refletividade. O dado sintético do Marmousi2 é composto pelas informações de densidade e velocidade de onde é possível calcular o perfil de refletividades usando as informações de densidade e velocidade.

Na Figura 5.1, é possível observar uma janela do Marmousi2 com a informação de velocidade, densidade e o perfil de refletividades calculado.

Para gerar as amplitudes sísmicas do Marmousi2, é necessário fazer a convolução do perfil de refletividades obtido com uma wavelet conhecida. O resultado desse processo de convolução com uma wavelet Ricker de frequência $30 \mathrm{~Hz}$ gera o perfil de amplitudes sísmicas que pode ser observado na Figura 5.2 .

A aplicação do método de deconvolução proposto nesse trabalho tem por objetivo fazer o caminho inverso do processo de obtenção da amplitude sísmica do marmousi. O objetivo é estimar os dois componentes que deram origem ao perfil de amplitudes.

O teste em dados sintéticos é especialmente relevante para os métodos de deconvolução pois é possível fazer uma comparação dos componentes da refletividade e wavelet reais, aqueles que deram origem ao perfil de amplitudes, com os obtidos pelo método proposto neste trabalho.

Com objetivo de demonstrar o resultado e o comportamento do método de deconvolução proposto, foram feitos três testes variando a condição das wavelets usadas. Dessa forma é possível observar como esses parâmetros influenciam os resultados. Outro parâmetro necessário para o método de deconvolução é o $\lambda$, relacionado a equação do Lasso, que para os testes no dado sintético é 0.001. Para cada teste será exibido a estimativa dos dois componentes da deconvolução, sendo que para melhor observação do resultado no perfil de refletividades estimados, uma área onde os refletores estão pouco espaçados é salientada.

Os parâmetros dos 3 testes propostos estão ressaltado nas tabelas 5.1, 5.2 e 5.3 .

As figuras 5.3, 5.4 e 5.5 mostram a comparação das wavelets dadas como parâmetro de entrada e a estimada pelo teste, assim como o perfil de refletividades estimado para cada teste. 

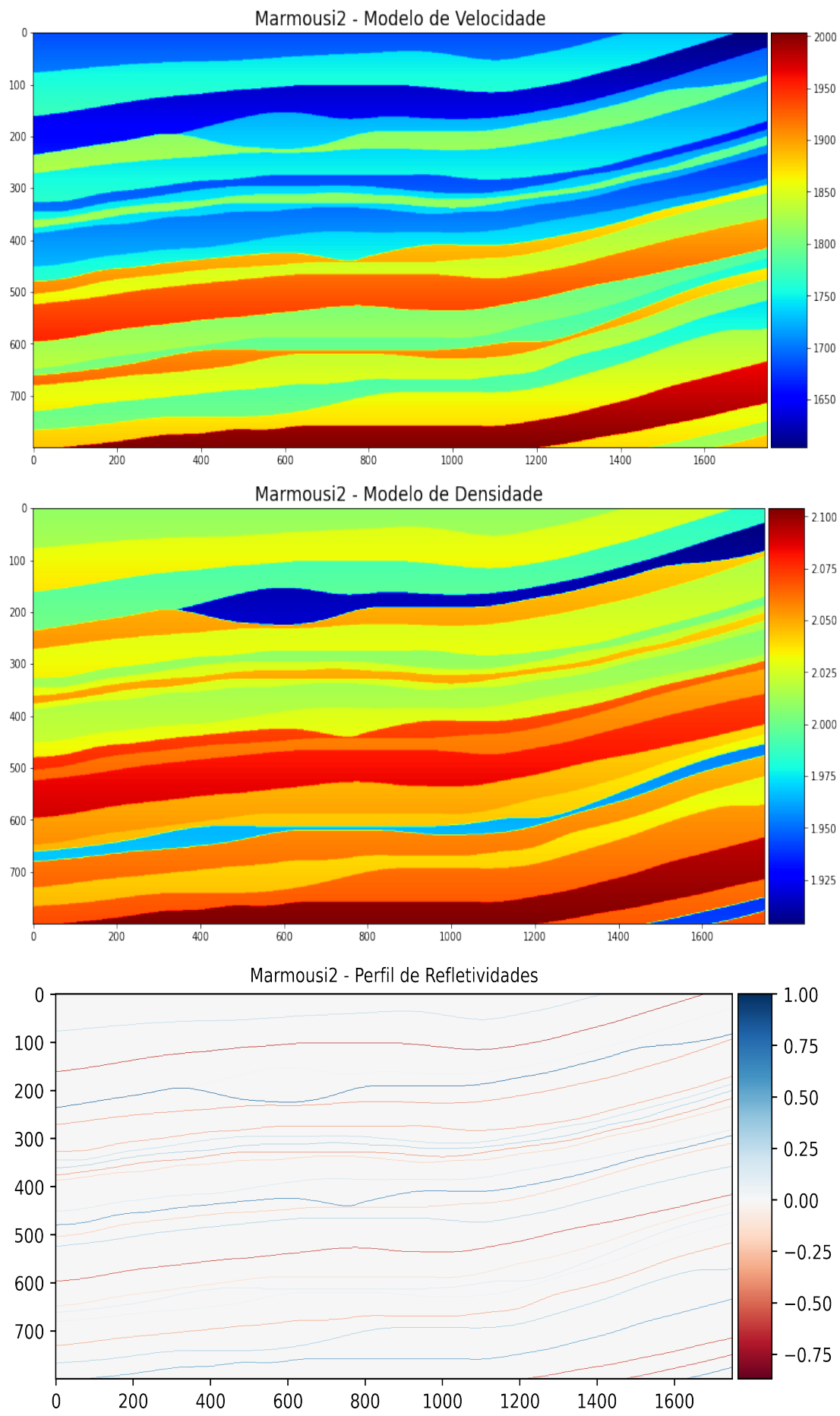

Figura 5.1: Perfil de refletividades calculado.

Finalmente, a Tabela 5.4 apresenta os resultados da similaridade, para cada componente estimado pelo método de deconvolução, como descrito no Capítulo 4. 


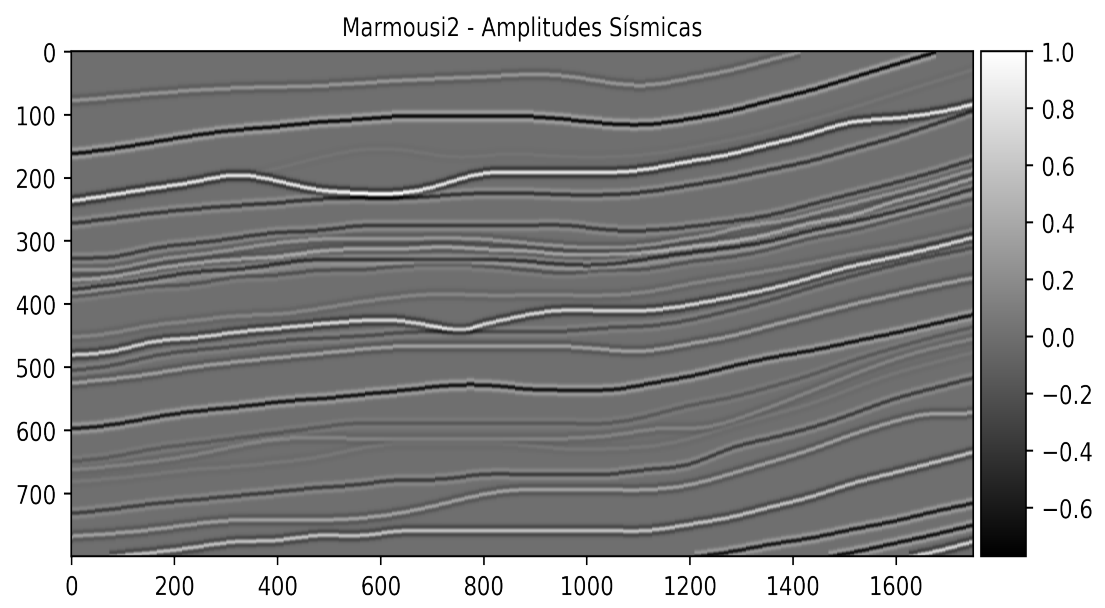

Figura 5.2: Amplitudes sísmicas obtidas pela convolução do perfil de refletividades com uma wavelet Ricker de frequência igual a $30 \mathrm{~Hz}$.

\begin{tabular}{l|l|l} 
& Wavelet Original & Wavelet Inicial \\
\hline \hline Tipo & Ricker & Ricker \\
Frequência(s) & $30 \mathrm{~Hz}$ & $30 \mathrm{~Hz}$ \\
Rotação de Fase & $30^{\underline{o}}$ & $0^{\underline{o}}$ \\
Comprimento da Onda & $0.2 \mathrm{~s}$ & $0.2 \mathrm{~s}$ \\
Taxa de Amostragem & $0.002 \mathrm{~s}$ & $0.002 \mathrm{~s}$
\end{tabular}

Tabela 5.1: Parâmetros teste 1.

\begin{tabular}{l|l|l} 
& Wavelet Original & Wavelet Inicial \\
\hline \hline Tipo & Ormsby & Ricker \\
Frequência(s) & $10,20,30,40 \mathrm{~Hz}$ & $30 \mathrm{~Hz}$ \\
Rotação de Fase & $30^{-}$ & $0^{\mathrm{o}}$ \\
Comprimento da Onda & $0.2 \mathrm{~s}$ & $0.2 \mathrm{~s}$ \\
Taxa de Amostragem & $0.002 \mathrm{~s}$ & $0.002 \mathrm{~s}$
\end{tabular}

Tabela 5.2: Parâmetros teste 2.

\begin{tabular}{l|l|l} 
& Wavelet Original & Wavelet Inicial \\
\hline \hline Tipo & Ormsby & Ricker \\
Frequência(s) & $10,20,30,40 \mathrm{~Hz}$ & $30 \mathrm{~Hz}$ \\
Rotação de Fase & $30^{-}$ & $0^{\mathrm{o}}$ \\
Comprimento da Onda & $0.2 \mathrm{~s}$ & $0.4 \mathrm{~s}$ \\
Taxa de Amostragem & $0.002 \mathrm{~s}$ & $0.002 \mathrm{~s}$
\end{tabular}

Tabela 5.3: Parâmetros teste 3.

\begin{tabular}{l|c|c|c} 
& Teste 1 & Teste 2 & Teste 3 \\
\hline \hline Similaridade Refletividades & 0.9597 & 0.9103 & 0.9084 \\
Similaridade Wavelets & 0.9995 & 0.9950 & 0.9931
\end{tabular}

Tabela 5.4: Resultados de similaridade calculados. 


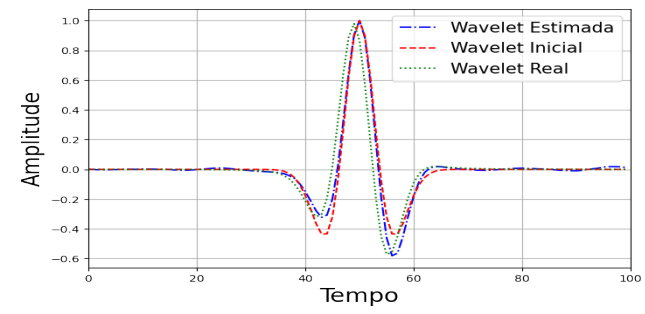

(a) Comparação das Wavelets.

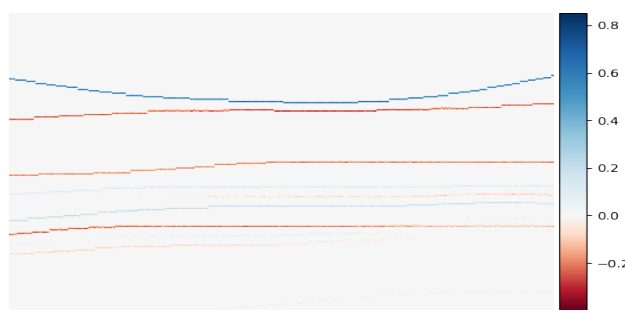

(b) Porção aproximada das refletividades.

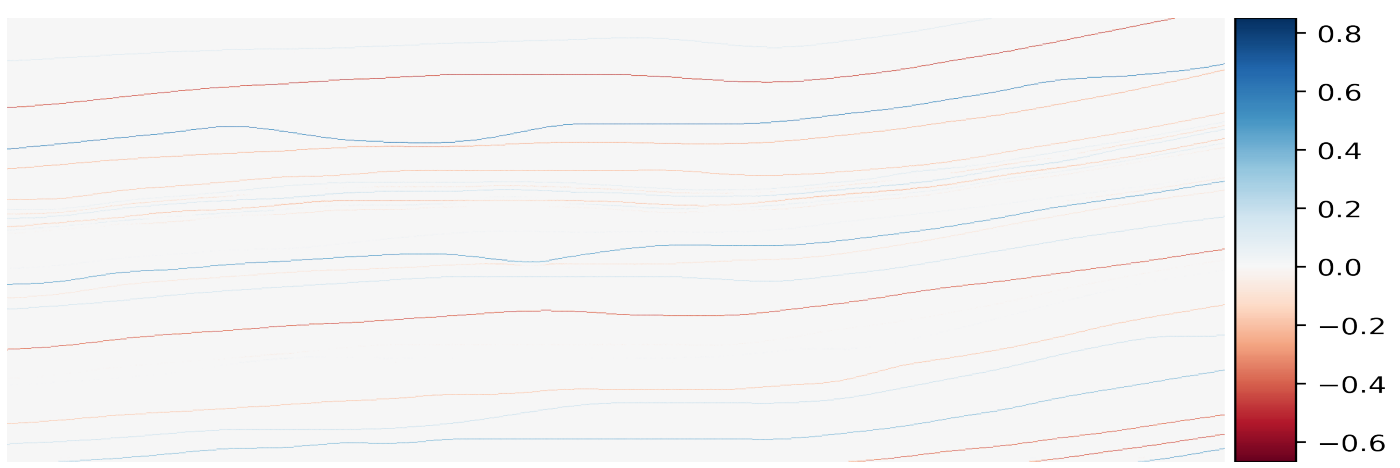

(c) Perfil de refletividades estimado.

Figura 5.3: Resultados apresentados do teste 1.

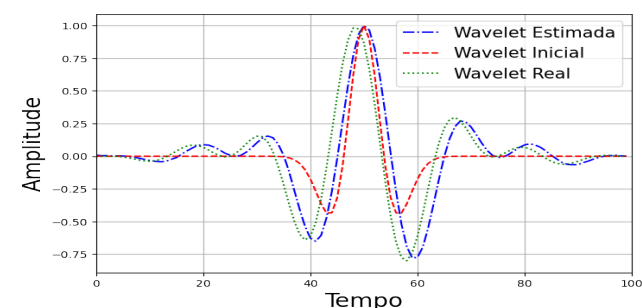

(a) Comparação das Wavelets.

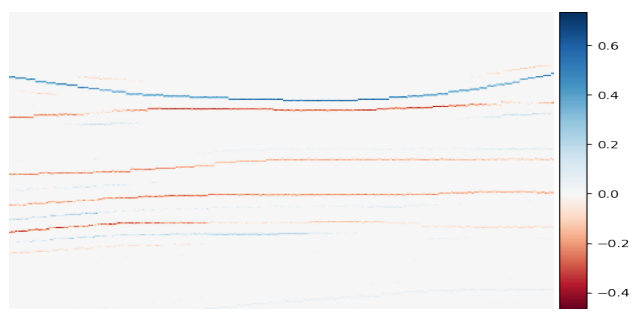

(b) Porção aproximada das refletividades.

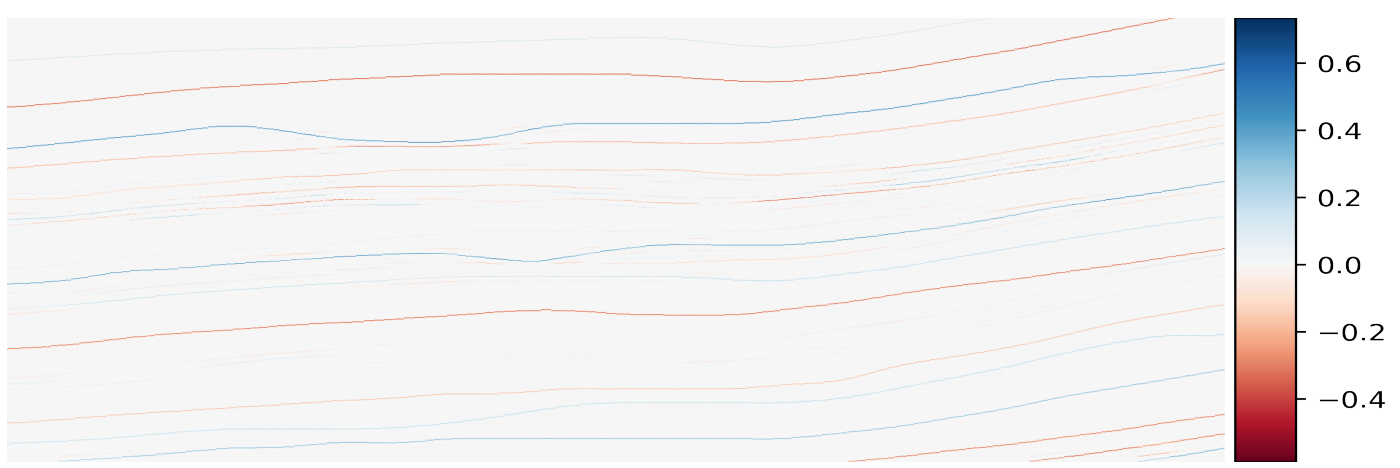

(c) Perfil de refletividades estimado.

Figura 5.4: Resultados apresentados do teste 2.

\section{2}

\section{Dado Real - Netherlands Offshore F3 Block}

Para demonstrar o comportamento do método em uma situação real, onde apenas a informação da amplitude sísmica está disponível e se deseja conhecer os componentes da refletividade e onda geradora, este trabalho usa o 


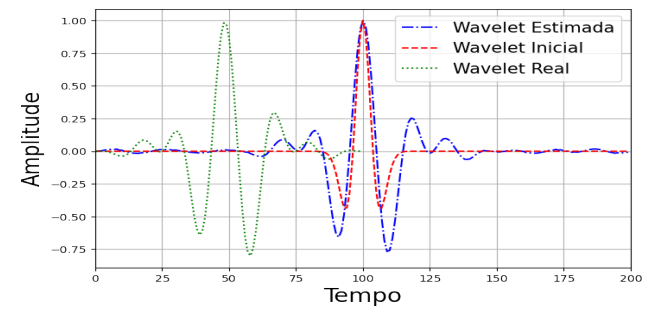

(a) Comparação das Wavelets.

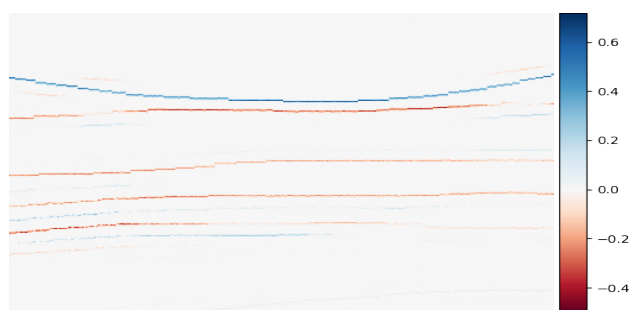

(b) Porção aproximada das refletividades.

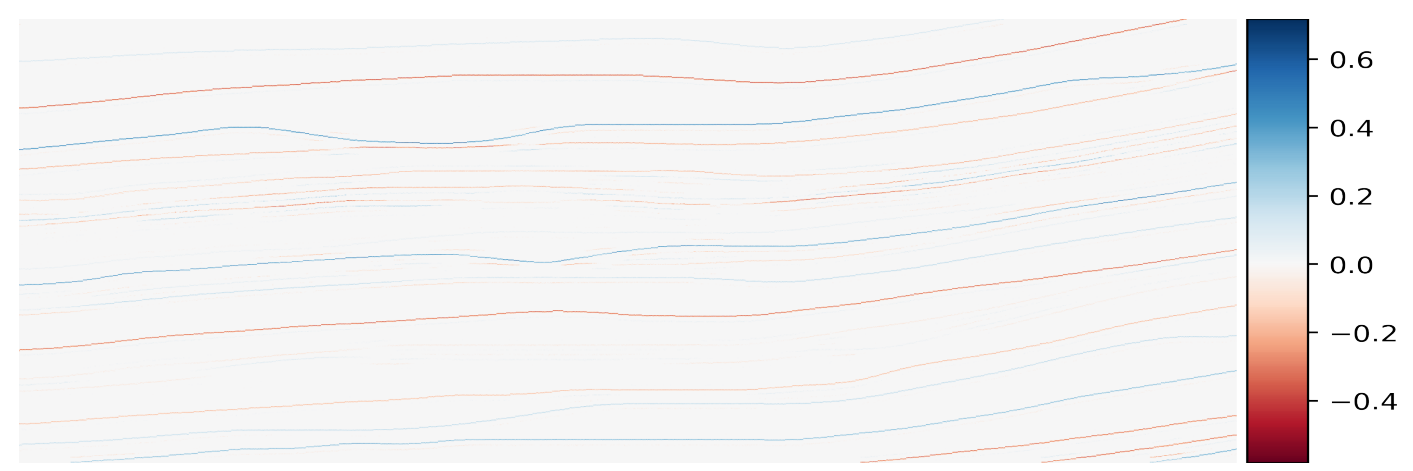

(c) Perfil de refletividades estimado.

Figura 5.5: Resultados apresentados do teste 3.

dado público Netherlands Offshore F3 Block para fazer a deconvolução. Esta aquisição 3D foi feita na costa da Holanda e é conhecido pela qualidade da aquisição e das informações associadas.

Para executar o teste, é selecionada uma inline desse bloco. A wavelet inicial escolhida foi uma gaussiana de desvio padrão $(\sigma)$ igual a 7 e 220 amostras no tempo, dessa forma podemos visualizar a capacidade do método de estimar a onda geradora deste bloco partindo de um ponto neutro. O parâmetro do Lasso foi $\lambda=10^{-4}$

A deconvolução foi executada por 20 iterações e os resultados podem ser observados na Figura 5.6.

\section{3}

\section{Discussão}

\section{Testes Dado Sintético:}

Os testes exibidos na seção anterior mostram a capacidade do método proposto de recuperar o componente de refletividades assim como a onda geradora do perfil de amplitudes sísmicas fornecido como entrada. O teste no dado síntético, como dito anteriormente, foi feito com objetivo de propor uma situação controlada onde se conhece previamente as características de geração do perfil de amplitude sísmica.

O três testes demonstram a capacidade do método de recuperar a wavelet de origem de forma satisfatória, alcançando a métrica usada, baseada na 


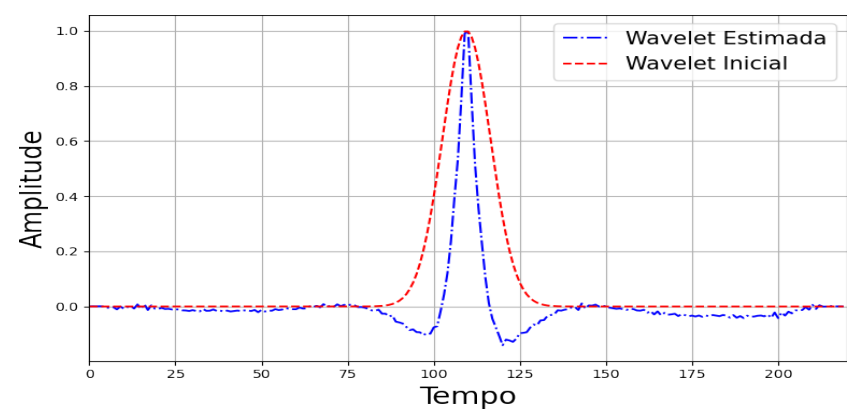

(a) Comparação das Wavelets.

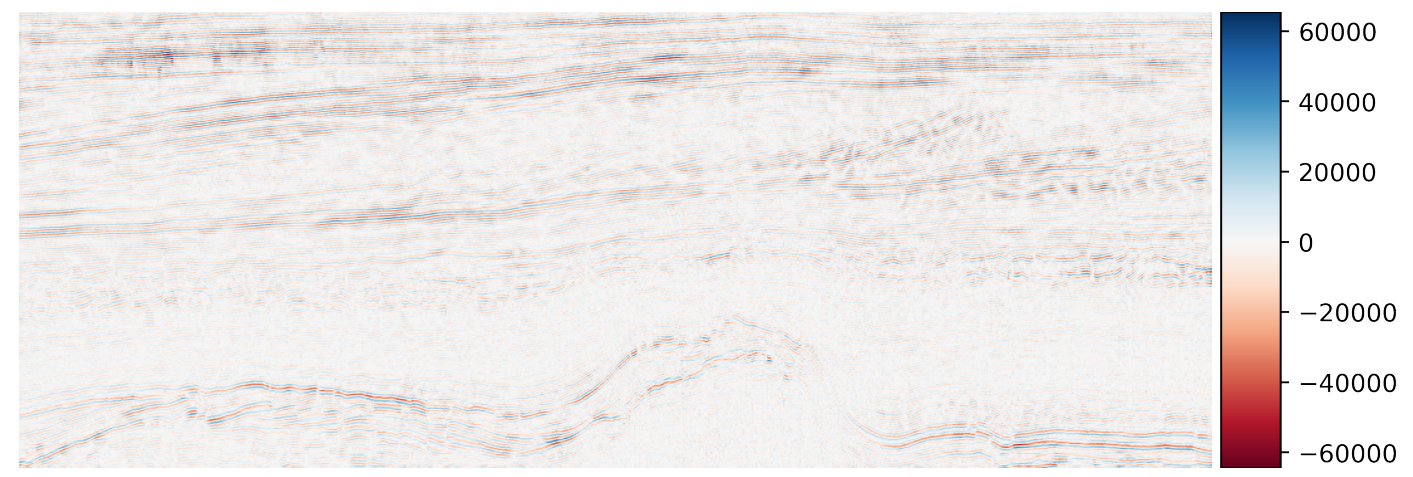

(b) Perfil de refletividades estimado.

Figura 5.6: Resultados da deconvolução da inline 260 do F3.

similaridade do cosseno, superior a 0.9 . Apesar da métrica representar uma similaridade alta, quando a wavelet geradora tem um deslocamento no eixo do tempo por causa de uma rotação de fase, esse deslocamento não parece ser observado na wavelet estimada. Uma suspeita é que esse deslocamento no tempo é tão sutil, que o método não parece considerá-lo. Por esse motivo as wavelets do Teste 1 e Teste 2 não estão exatamente sobrepostas, porém o deslocamento estimado que resulta nos maiores resultados de similaridade para o Teste 1 e 2 são, respectivamente, -1 e -2 amostras no eixo do tempo.

O Teste 3 demonstra de forma mais específica que esse deslocamento no tempo não influencia de forma significativa no resultado da refletividade ou no formato da onda estimada. Neste teste, propositalmente, a wavelet real e a inicial não compartilham da mesma amostragem no tempo. Essa discrepância além de simular a falta de conhecimento do comprimento ideal da onda em uma situação real, onde essa informação é desconhecida, mostra que a forma da onda é o ponto principal na estimação da wavelet. Para esse teste, o resultado da métrica de similaridade alcançou 0.99.

Em relação as refletividades estimadas, os três testes mostram a capacidade do método de atingir uma solução esparsa das refletividades por traço e um perfil de refletividades muito próximo ao original. Nas figuras de observação dos resultados, também é destacado uma porção do dado onde espera-se que seja a parte mais difícil para o método recuperar os refletores, devido ao 
pouco espaçamento e consequentemente a mistura dos sinais após o processo convolucional. No Teste 1 especificamente, os refletores na porção aproximada das refletividades parecem estar melhor definidos que nos outros dois testes, provavelmente porque a wavelet inicial é mais próxima da wavelet original. Apesar disso, o método parece estimar de forma bem acurada os refletores mais importantes, de resposta mais intensa. Os refletores menos de resposta menos intensa, apesar do método conseguir recuperá-los, não apresentam a mesma continuidade dos mais intensos.

Tendo essa limitação em vista, o método ainda obteve a resposta da similaridade cosseno calculada acima de 0.90, o que indica que o as refletividades recuperadas para os testes são próximas do perfil de refletividades real.

\section{Teste Dado de Campo}

O teste no dado de campo mostra alguns pontos interessantes sobre o a aplicação do método em um dado real. Primeiramente, o resultado dos refletores estimados segue os pontos positivos e limitações observados nos testes no dado sintético. Os refletores de resposta mais intensa são mais bem delineados e também é possível observar a recuperação razoável dos refletores pouco espaçados.

É conhecido que no dado do F3 existe uma rocha formada por um composto de sal, que resulta em um refletor muito proeminente. No resultado das refletividades estimado, esse refletor está muito bem demarcado, o que é importante pelo tipo de rocha e pelo que ele pode representar para a indústria de prospecção de hidrocarbonetos.

Porém o resultado mais interessante deste teste foi a capacidade de ressaltar as descontinuidades geradas pelas falhas. É possível observar esse detalhe na imagem aproximada da Figura 5.7.

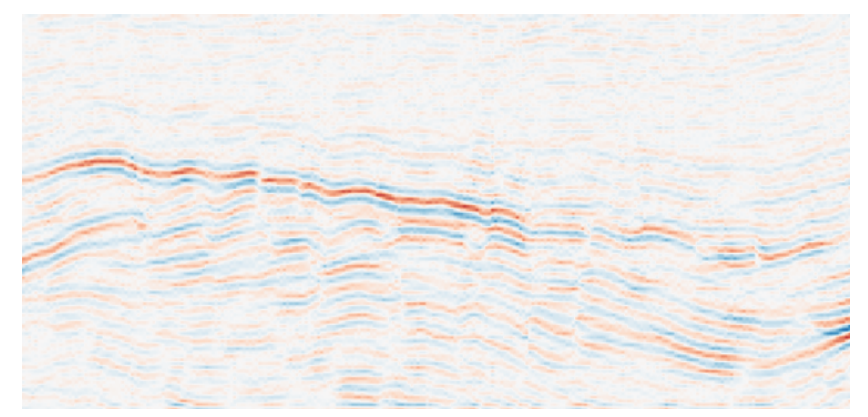

Figura 5.7: Figura ressaltando as descontinuidades criadas por falhas sísmicas que podem ser observadas mais claramente no perfil de refletividades estimado.

Também é interessante observar o formato da wavelet estimada que, partindo de um ponto neutro, se aproxima de um formato mais próximo de uma representação teórica de uma wavelet geradora do sinal sísmico. 
Por fim, como ressaltado no Capítulo 3, o método foi paralelizado em CPU, fazendo o uso dos diversos núcleos de processamento disponíveis. O teste foi executado em uma CPU de modelo Ryzen 3600 - 6 núcleos e a comparação do tempo de execução do método pode ser observado na tabela 5.5.

\begin{tabular}{l|l|l} 
& 1 Iteração & 5 Iterações \\
\hline \hline Teste Sequencial & $955 \mathrm{~s}$ & $4995 \mathrm{~s}$ \\
Teste Paralelo & $160 \mathrm{~s}$ & $830 \mathrm{~s}$
\end{tabular}

Tabela 5.5: Comparação do tempo de execução da deconvolução em sequencial e paralelo

Pode-se perceber que a diferença do tempo de processamento é muito significativa, cerca de 6 vezes mais rápido quando executado em paralelo. Tendo em vista o contexto das dimensões dos dados sísmicos disponíveis, métodos paralelizáveis são extremamente importantes para o emprego de um método deconvolucional porque permitem o uso de forma mais eficiente do recurso computacional disponível.

Por fim, na Figura 5.8 mostra o gráfico do erro estimado por iteração, como descrito no Capítulo 4, para este teste. É possível perceber a clara diminuição do erro até um ponto onde as soluções de cada nova iteração diminuem ou aumentam pouco a medida do erro calculada, assinalando uma convergência para soluções próximas.

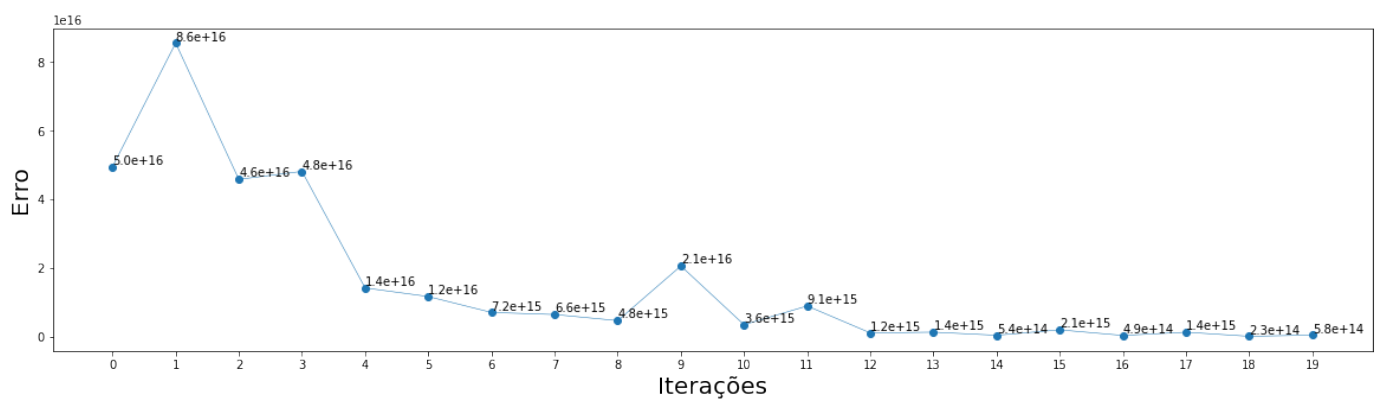

Figura 5.8: Gráfico da medida de erro por iteração. 


\section{6}

\section{Conclusões e Trabalhos Futuros}

Durante o desenvolvimento deste trabalho, foi discutido o problema da inversão de um perfil de amplitudes sísmicas com objetivo de recuperar os dois componentes de um processo convolucional, a onda geradora e o perfil de refletividades. Ao fazer um estudo sobre a literatura da área neste problema, é perceptível o número de trabalhos que partem da suposição que este perfil de refletividades tem uma característica esparsa. Aproveitando esta suposição, diversos métodos usam a rica literatura da modelagem esparsa e recuperação do sinal esparso como suporte para propor uma solução eficiente.

Assim como esses outros autores, este trabalho também se baseia nos princípios da modelagem esparsa. Usamos o algoritmo de regressão LASSO para primeiramente recuperar o vetor esparso que representa os picos de refletividade associados a um traço sísmico, como na 3.1. Partindo dessa estimativa das refletividaes do traço, é possível estimar também a onda geradora associada ao traço resolvendo um simples sistema linear. Por fim, usamos a média das ondas estimadas traço a traço para obter uma única resposta da wavelet associada. A decisão de trabalhar traço a traço, em oposição ao um conjunto de traços simultaneamente, que parece ser o caminho mais explorado nos outros trabalhos, permite que estes subproblemas sejam resolvidos independentemente. Aproveitando desta modelagem do problema, usamos uma biblioteca que permite paralelizar o processamento destes subproblemas em diversas threds de execução ao mesmo tempo e também divide para os diversos núcleos da CPU usada. Usando este artifício, conseguimos reduzir consideravelmente o tempo de execução do método, cerca de 6 vezes mais rápido (Ryzen 3600 6 núcleos), o que é bem significativo para área de geofísica que trabalha com uma quantidade muito grande de dados.

Após a proposta do método, este trabalho também discute uma melhor forma de analisar o resultado do processo de deconvolução. Uma medida da similaridade que avalia os dois componentes estimados com o ground truth é necessária para medir a qualidade do processo proposto e também a influência dos parâmetros. Desta forma, as duas medidas de similaridade usadas para avaliar cada componente foram baseadas na similaridade do cosseno, também usada em outros trabalhos [Fernandes, Lopes e Gattass, 2017]. Para usar de 
forma apropriada essa medida, transformamos o vetor esparso da refletvidade para um vetor denso usando um filtro gaussiano, desta forma a medida pode ser calculada ponto a ponto, criando uma certa robustez a pequenos deslocamentos dos picos da refletividade porém, ainda levando em consideração a intensidade de tais picos. Similarmente, percebemos que a similaridade do cosseno não representava de forma acurada a distancia entre a wavelet original e a estimada por causa de diferenças no comprimento da onda ou leves deslocamentos causados pelo próprio método de deconvolução, apesar do formato da onda ser praticamente igual. Para contornar essa diferença observada, a medida de similaridade é calculada fazendo o deslocamento de uma onda sobre a outra, onde a maior similaridade encontrada é considerada em companhia com o descolamento necessário.

Por fim, este trabalho avalia seus resultados usando um conjunto de dados sintético, onde todas as variáveis são conhecidas e a avaliação pode ser feita de forma objetiva, e em um dataset de um cubo sísmico 3D. Nossos testes no dado sintético mostram a capacidade do método de recuperar tanto a wavelet geradora como o perfil de refletividades de forma acurada levando em consideração a fase da wavelet assim como de naturezas diferentes, Ricker e Ormsby. Os testes no dado real de uma inline do cubo 3D, mostram um resultado também interessante para recuperação das refletividades, os horizontes parecem ser, em sua maioria, claros e bem definidos, principalmente os horizontes que correspondem os picos de maior contraste de impedância. Também é notável que a wavelet estimada para este teste, partindo inicialmente de uma curva normal, onde não existem valores negativos, se aproxima da forma de uma wavelet conhecida teoricamente. Os testes nos dois datasets utilizados também elucidam as vantagens em tempo de execução ao dividir as tarefas entre múltiplas threds que são executadas em paralelo na CPU.

Como resultado desta dissertação, uma versão deste trabalho foi submetido e aceito no evento Society of Exploration Geophysicists (SEG) Annual Meeting 2020 para modalidade de apresentação oral.

\section{1}

\section{Trabalhos Futuros}

- Um dos pontos evidentes para o aprimoramento do processo de deconvolução proposto é tentar modelar os efeitos de reflexão e absorsão da onda geradora durente o trânsito no meio rochoso, como faz [Sui e Jianwei, 2019]. É esperado que esse tipo de abordagem consiga melhorar a aproximação da wavelet ao considerar um comportamento não estacionário o que também influenciaria a estimação dos refletores 
mais profundos, que sofrem mais com esse efeito.

- Uma das melhorias que possivelmente poderiam facilitar o acesso a esse método é fazer a execução em GPU, diminuindo o tempo de execução significativamente. Desta forma, a aplicação em modelos 3D pode ser muito mais eficiente em tempo de processamento. 


\section{Referências bibliográficas}

[Andrade, Gattass e Fernandes, 2020] ANDRADE, F.; GATTASS, M. ; FERNAN-

DES, R.. Lawie - sparse-spike deconvolution with lasso and wiener filter. 2020.

[Beck e Teboulle, 2009] BECK, A.; TEBOULLE, M.. A fast iterative shrinkage-thresholding algorithm for linear inverse problems. SIAM journal on imaging sciences, 2(1):183-202, 2009.

[Dumitrescu e Irofti, 2018] DUMITRESCU, B.; IROFTI, P.. Dictionary Learning Algorithms and Applications. Springer International Publishing AG, 2018.

[Elad, 2010] ELAD, M.. Sparse and Redundant Representations. From Theory to Applications in Signal and Image Processing. SpringerVerlag New York, 1 edition, 2010.

[Fernandes, 2019] FERNANDES, R. C.. Modelagem Esparsa e Supertraços para Deconvolução e Inversão Sísmicas. $\mathrm{PhD}$ thesis, Pontifícia Universidade Católica do Rio de Janeiro, 2019.

[Fernandes, Lopes e Gattass, 2017] FERNANDES, R.; LOPES, H. ; GATTASS, M.. Lobbes: An algorithm for sparse-spike deconvolution. IEEE Geoscience and Remote Sensing Letters, 14(12):2240-2244, 2017.

[Kaaresen e Taxt, 1998] KAARESEN, K. F.; TAXT, T.. Multichannel blind deconvolution of seismic signals. Geophysics, 63(6):2093-2107, 1998.

[Kibangou e Favier, 2007] KIBANGOU, A. Y.; FAVIER, G.. Toeplitzvandermonde matrix factorization with application to parameter estimation of wiener-hammerstein systems. IEEE Signal Processing Letters, 14(2):141-144, 2007.

[Kormylo e Mendel, 1978] KORMYLO, J.; MENDEL, J. M.. On maximumlikelihood detection and estimation of reflection coefficients. Technical report, UNIVERSITY OF SOUTHERN CALIFORNIA LOS ANGELES DEPT OF ELECTRICAL ENGINEERING, 1978. 
[Margrave e Henley, 2011] MARGRAVE, G. F.; LAMOUREUX, M. P. ; HENLEY, D. C.. Gabor deconvolution: Estimating reflectivity by nonstationary deconvolution of seismic data. Geophysics, 76(3):W15-W30, 2011.

[Martin, Wiley e Marfurt, 2006] MARTIN, G. S.; WILEY, R. ; MARFURT, K. J.. Marmousi2: An elastic upgrade for marmousi. The Leading Edge, 25(2):156-166, 2006.

[Pedregosa et al., 2009] PEDREgOSA, F.; VAROQUAUX, G.; GRAMFORT, A.; MICHEL, V.; THIRION, B.; GRISEL, O.; BLONDEL, M.; PRETTENHOFER, P.; WEISS, R.; DUBOURG, V.; VANDERPLAS, J.; PASSOS, A.; COURNAPEAU, D.; BRUCHER, M.; PERROT, M. ; DUCHESNAY, E.. Scikit-learn: Machine learning in Python. Journal of Machine Learning Research, 12:2825-2830, 2011.

[Rish e Grabarnik, 2015] RISH, I.; GRABARNIK, G. Y.. Sparse Modeling. CRC Press, 2015.

[Rosa, 2010] ROSA, A. L. R.. Análise do Sinal Sismico, volumen 1. Sociedade Brasileira de Geofísica (SBGf), 2010.

[Sui e Jianwei, 2019] SUI, Y.; MA, J.. A nonstationary sparse spike deconvolution with anelastic attenuation. Geophysics, 84(2):R221-R234, 2019.

[Tibshirani, 1996] TIBSHIRANI, R.. Regression shrinkage and selection via the lasso. Journal of the Royal Statistical Society: Series B (Methodological), 58(1):267-288, 1996.

[Varoquaux e Grisel 2009] VAROQUAUX, G.; GRISEL, O.. Joblib: running python function as pipeline jobs. packages. python. org/joblib, 2009.

[Velis, 2008] VELIS, D. R.. Stochastic sparse-spike deconvolution. Geophysics, 73(1):R1-R9, 2008.

[Walt e Gael, 2011] VAN DER WALT, S.; COLBERT, S. C. ; VAROQUAUX, G.. The numpy array: a structure for efficient numerical computation. Computing in Science \& Engineering, 13(2):22, 2011.

[Wang et al., 2016] WANG, L.; ZHAO, Q.; GAO, J.; XU, Z.; FEHLER, M. ; JIANG, X.. Seismic sparse-spike deconvolution via toeplitz-sparse matrix factorization. Geophysics, 81(3):V169-V182, 2016. 
[Wiener, 1964] WIENER, N.. Extrapolation, interpolation, and smoothing of stationary time series. The MIT press, 1964. 